\title{
Atypical Presentation of Urinary Schistosomiasis
}

\author{
Sunayana M. Jangla ${ }^{1}$, Susan Cherian ${ }^{1}$, Bhupesh S. Machhi ${ }^{1}$, Anita N. Gadgil ${ }^{2}$ \\ ${ }^{1}$ Department of Pathology, Microbiology Section, Bhabha Atomic Research Centre Hospital, Anushakti Nagar, \\ Trombay, Mumbai, India; \\ ${ }^{2}$ Department of Surgery, Bhabha Atomic Research Centre Hospital, Anushakti Nagar, Trombay, Mumbai, India
}

\begin{abstract}
Schistosomiasis or Bilharziasis is a water-borne parasitic disease which affects different organs depending on species involved. Urinary schistosomiasis is diagnosed mainly by the presence of urinary complaints, urinary excretion of parasite eggs and their presence in bladder as seen on histopathology along with changes on cystoscopy. We present a case of an asymptomatic adult Indian female with long standing history of passing adult worms in urine but lack of eggs. The worms were identified as Schistosoma and the patient was treated with Praziquantel. She came from an area which is not known to be endemic for Schistosoma. J Microbiol Infect Dis 2019; 9(2):95-99.
\end{abstract}

Keywords: Asymptomatic, Atypical, Non-endemic Area, Schistosoma

\section{INTRODUCTION}

Schistosomiasis, which is a water-borne parasitic disease, affects 200 million people and 600 million people in more than 76 countries are at risk from it [1]. In the tropical and subtropical countries, it is endemic and seen mainly in Africa and eastern Mediterranean region [1]. In Asian countries, Schistosoma japonicum, Schistosoma mekongi and Schistosoma malayensis are prevalent. Schistosoma haematobium is among those species which are uncommon in Asia [2]. Although Schistosomiasis is known to be a disease of the poor and rural population, there has been a significant change in its geographic distribution. The reasons attributed to this are population movement from endemic zones, increase in travel for various purposes and expansion of natural habitat of snail due to new irrigation projects. This has given rise to newer endemic foci [2]. Although India has been considered as a non-endemic country for human schistosomiasis, areas of Gimvi District of Maharashtra, Madurai district of Madras and Raipur district of Madhya Pradesh have been discovered as endemic foci [2] and sporadic cases of human schistosomiasis from India also exist [2]. This may be because of coexistence of various species giving rise to newer hybrid strains pathogenic to man [2]. Most human diseases are caused by $S$. haematobium which involves the bladder, S. mansoni which involves intestines and $S$. japonicum which is confined to intestinal tract and liver [3]. It is diagnosed mainly by presence of eggs in urine and or stool of the patient. We present a case of an adult Indian female with long standing history of passing adult Schistosoma worms in urine, was mostly asymptomatic and her stool and urine did not show Schistosoma eggs except on one occasion nor did her cystoscopy or any other investigation showed significant findings. Moreover, she came from an area which is not known to be endemic for Schistosoma.

\section{CASE REPORT}

A 51-year old female, a home-maker and a mother of two adults, presented with history of passing live worms and blood strand in the urine since two and half years. She generally passed three to four worms at a time in the morning with a frequency of two-three such episodes in a month. Before passage of worms, every time she experienced crampy pain in lower abdomen. She passed them on a flat surface and each worm appeared as a blood drop which later started uncoiling and moving.

Correspondence: Dr. Sunayana Jangla, Microbiology Section, Department of Pathology, Jangla, Bhabha Atomic Research Center Hospital, Trombay, Mumbai, Maharashtra, India E-mail: sunayanajangla79@gmail.com

Received: 13 February 2019 Accepted: 11 April 2019

Copyright (C JMID / Journal of Microbiology and Infectious Diseases 2019, All rights reserved 
There was no history of fever, bowel complaints or urinary complaints like dysuria, hematuria, increased frequency of urination or itching or redness in a particular body area. She also gave history of irregular, heavy and long-lasting menstrual periods since two and a half years. The urinary worms were identified as nematode in another institute and she was given a single 400 milligrams dose of Albendazole orally. A year later from her first complaint, she developed pain in back and lower abdomen localized to the flank. Nine months later she developed rash with itching, loss of taste sensation and decreased smell sensation.

Five months later, she developed extreme fatigability and her menstrual irregularities were still persistent. During this period, she was given other anthelminthics like Ivermectin, Pyrantel Palmoate and Heterazan due to nonresponsiveness to Albendazole. She was a known case of hypothyroidism and hypertension on medication. She comes originally from Orissa but is settled in Mumbai since the past thirty years. She gave history of frequent visits to beaches in Orissa and Mumbai before showing the above symptoms. There was no history of travel to African or Central and South American countries. There was no history of contact with animals, having pets or any family member with similar complaints. Consumption of nonvegetarian food, mostly fish was once a week.

In the beginning of her complaints two and half years back, her hemoglobin was $8.1 \mathrm{~g} / \mathrm{dl}$. At that time, her urine and stool did not show presence of eggs, cysts or other significant findings. Her blood eosinophil count was also normal. All her blood investigations were received on several occasions thereafter and were normal except haemoglobin which was persistently low. Her urine and stool samples received on several occasions were also normal except on one occasion a year later from her first complaint, when there were 15-20 pus cells and 20-30 red blood cells per high power field in urine and stool showed presence of occult blood. During this course, she had also submitted her urine and stool samples for examination to another institute but was normal. Cystoscopy was done fifteen days after her first complaint, that is two and half years back which showed trigonitis and whitish nodule in inter-sphincteric area.
Cystoscopic biopsy done then showed metaplastic squamous epithelium negative for malignancy.

Pelvic Ultrasonography (USG) revealed leiomyoma of the uterus. A year later, cystoscopy was repeated which showed thread like whitish structure present submucosally in right mid-posterior calyx in right and left kidney. No other significant findings were present especially in the bladder. Computerized Tomography scans of the abdomen, bilateral retrograde pyelography and flexi-ureteroscopy were normal. She underwent hysterectomy with salphingo-oophorectomy based on her USG report and was given hematinics due to which her hemoglobin reached to $12 \mathrm{~g} / \mathrm{dl}$. She discontinued the anthelminthic drugs due to metallic taste in the mouth and inability to eat.

On her current visit to our hospital, that is 30 months after her first complaints, all her blood investigations and stool microscopy results were normal. On gross examination, the urine sample received was pale yellow, clear and contained three worms. All three appeared similar. Each was motile, pinkish-brown in color and 1.5 to 2 centimeters in length (Figure 1).

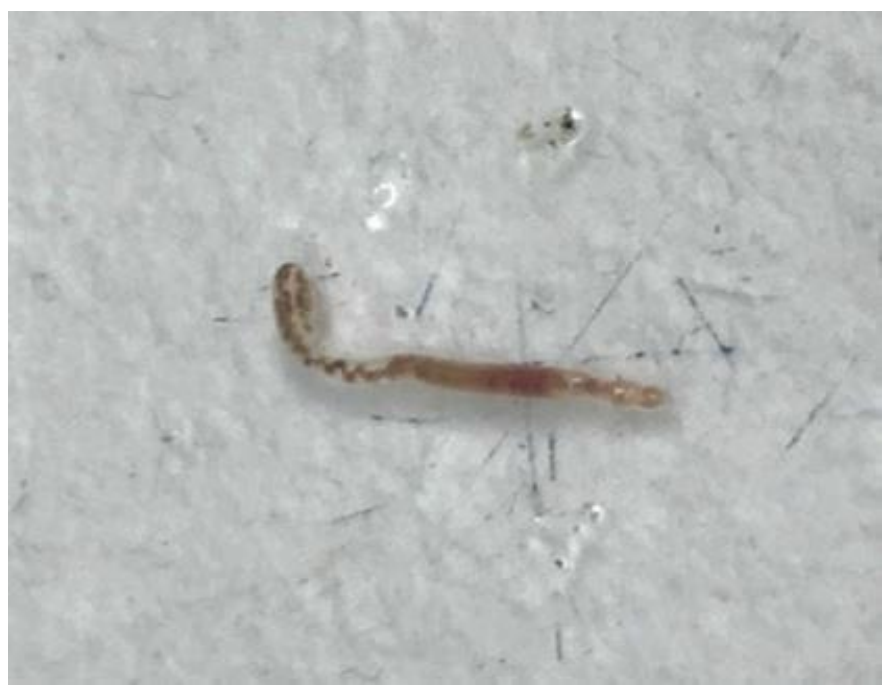

Figure 1: Worm on gross examination.

Under high power lens, it showed two different ends. One end showed a structure resembling a sucker (Figure 2) and other end was blunt. Her urine was centrifuged and the sediment showed a single egg with a well-defined outer and inner shell and a lateral spine and occasional pus cells and red blood cells (Figure 3 ). The patient 
also submitted another urine sample passed between 10 a.m. and 2 p.m. but it did not show eggs. A provisional identification of Schistosoma was given. The images of the worm were sent to Indian Society of Parasitology (ISP) and they confirmed it as female counterpart of Schistosoma. The patient was given praziquantel in dose of $40 \mathrm{mg} / \mathrm{kg}$ body weight in two divided doses. She has been called for follow-up after six weeks.

Under high power lens, it showed two different ends. One end showed a structure resembling a sucker (Figure 2) and other end was blunt. Her urine was centrifuged and the sediment showed a single egg with a well-defined outer and inner shell and a lateral spine and occasional pus cells and red blood cells (Figure 3 ). The patient also submitted another urine sample passed between 10 am and 2 pm but it did not show eggs. A provisional identification of Schistosoma was given. The images of the worm were sent to Indian Society of Parasitology (ISP) and they confirmed it as female counterpart of Schistosoma. The patient was given Praziquantel in dose of $40 \mathrm{mg} / \mathrm{kg}$ body weight in two divided doses. She has been called for follow-up after six weeks.

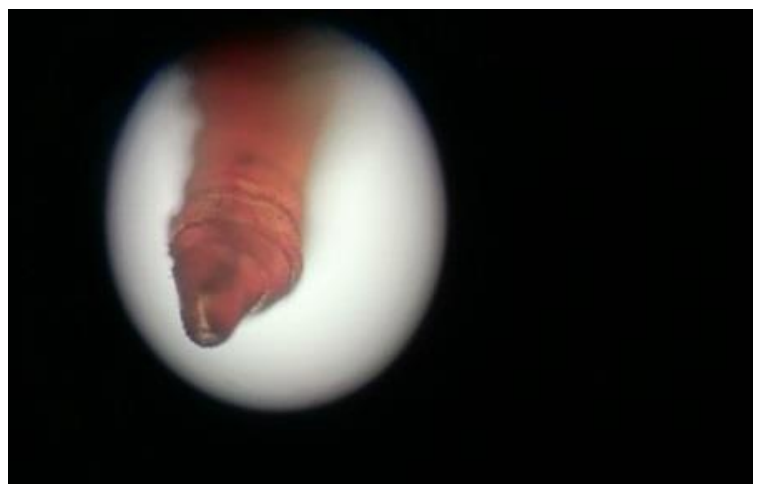

Fig.2: End with sucker as seen under 40x.

\section{DISCUSSION}

Schistosomiasis is endemic in various countries of different continents including some parts of India [3,4]. Upon contact with fresh water, parasite eggs hatch into miracidia which affects the snail. Snail is the intermediate host in which they transform into the infectious larval forms, the cercariae. On release into water, cercariae attach to humans and penetrate the skin. They lose the skin and become schistosomulae that enter the circulatory system. They migrate to portal blood in the liver and mature into adults and migrate to pelvic veins for oviposition [5]. Urinary schistosomiasis manifests as two forms, acute and chronic. Acute form or Katayama fever occurs three to nine weeks after infection and is characterized by fever, malaise, lymphadenopathy, splenomegaly, eosinophilia and dermatitis in area of penetration. Chronic form occurs two to seven months after infection and results due to presence of eggs in organs like bladder wall, ureters, and genitals along with inflammatory granulomatous reaction to it. It is characterized by hematuria, dysuria, increased frequency of micturition and lower back pain [5]. Diagnosis of urinary schistosomiasis is based on symptoms, eosinophilia, presence of proteinuria and Schistosoma eggs in the urine, serological tests like antibodies detection by ELISA and presence of urinary Eosinophil Cationic Protein (ECP). Abdominal X-Ray, USG and excretory urography are helpful at a later stage by identifying bladder wall calcifications, severe pathology and strictures and hydronephrosis respectively. Cystoscopy is indispensable especially when egg counts and signs and symptoms are inconclusive. Histopathology of the bladder biopsy can detect presence of egg and surrounding fibrosis and chronic inflammation [5]. Polymerase Chain Reaction (PCR) can be highly sensitive and specific [6]. In our case, the patient did not have any urinary symptoms. There was neither eosinophilia nor presence of protein or red blood cells in the urine except on one occasion. Eggs in urine were absent in all her samples except one. Finding ova in urine microscopy establishes a definitive diagnosis [7]. The paucity of ova in urine of our patient in spite of such a long standing history of passing worms is unusual. Due to low and sporadic urinary excretion rate of eggs, early diagnosis is always difficult and delayed $[6,8,9]$ as was seen in our case also. Absence of symptoms in our patient may be due to lack of eggs as the eggs are responsible for pathogenesis and not adult worms [10,6]. To avoid missing of eggs, the patient was asked to give urine sample between 10:00 am and 2:00 $\mathrm{pm}$ when there is a possibility of maximum egg load [6] and each time the urine sample was centrifuged $[3,6]$ but that did not help. Oranusi $\mathrm{CK}$ et al. observed that in chronic stages excretion of eggs is invariably absent and 
investigations like bladder biopsy and serology may be more specific [11]. In our case serology was not available and bladder biopsy was not repeated. Cystoscopy and bladder biopsy done initially showed signs of irritation but no egg deposition. Cystoscopy repeated a year later also showed a normal bladder and other radiological findings were also normal. Houmsou $R$ et al. found that observation of urine color helps in estimating the infection prevalence and correlates well with egg counts [12]. In our case also, the pale-yellow color of urine indicated a less severe infection and thus a low egg count. Rare cases of Schistosoma involving unusual sites without suggestive symptoms and absence of eggs in urine and stool have been reported in literature though histopathology of the affected organ in these cases showed presence of eggs [4,9-11]. Studies have shown the percentage of asymptomatic Schistosoma infection to be between 25 and $90 \%$ [13]. Another unusual finding in our case was the presence of a lateral spine in the egg which suggests that the species may not be $S$. haematobium as it's egg has a terminal spine [3].The morphological features of the egg suggest that it is of $S$. mansoni amongst the species that are known. $S$. mansoni causes intestinal and hepatosplenic infections. It is endemic in countries of South America, Africa, eastern Mediterranean and certain Caribbean islands. No cases have been reported from India so far [3]. Our patient did not have history of travel to these endemic places. However, there are various factors like endemic foci, sporadic cases, native snails serving as intermediate host and species interbreeding through which acquisition of infection with this organism may be possible [2]. Also, in a study by Meurs L, excretion of $S$. mansoni eggs via ectopic route that is urine was found in some patients who had mixed infections by $S$. haematobium as well as $S$. mansoni infections [14]. This ectopic egg excretion was found to be associated with lowest prevalence of bladder morbidity [14]. In our case also, there were no significant symptoms and bladder changes in spite of longstanding history of worm excretion. This could possibly be due to inefficiency of $S$. mansoni to lay eggs in and unusual site. Extrahepatic Schistosomiasis in the form of glomerulopathy can be caused by $S$. mansoni. These patients may have only minor urinary complaints like proteinuria or haematuria or severe manifestations like nephrotic syndrome [15]. In Asian countries, $S$. haematobium is less prevalent as compared to other species [2]. But several different Schistosome species of animals are prevalent in India and their coexistence may give rise to newer hybrid pathogenic strains [2]. Hence, another possibility is of infection by a new species. High proportions of presence of cercarial dermatitis in Indians from various places have been reported [16]. It is noteworthy that our patient has history of repeated exposures to beaches in Orissa and Mumbai which are not yet reported as endemic areas for Schistosoma. However, due to increase in travel and population migration these waters can get contaminated and even a single exposure to contaminated waters can cause infection [13]. But the species could not be confirmed as the required tets were not available with us. Bierman et al. observed that species identification may not always be possible due to decreased sensitivity of urine and stool microscopy in light infections and non-reliability of microscopy due to this reason [13]. Hence it was very important to confirm the worm identification. This helped in starting the appropriate treatment and highlighted the fact that although rare from non-endemic areas, possibility of infection with this parasite remains and the need for more sensitive and specific tests like PCR is essential. Patient was given oral Praziquantel which is the drug of choice in a dose of $40 \mathrm{mg} / \mathrm{kg}$ body weight in two divided doses $[1,3]$. She has been called for follow-up after six weeks. Bladder carcinoma is a common complication of chronic $S$. haematobium infection $[1,3]$. She will be regularly screened for it as there were squamous metaplastic changes in bladder and long-standing history of passing worms in urine.

Conclusion: This is an unusual case report for its asymptomatic form and atypical species especially from a non-endemic area. A high index of suspicion from the clinician and the laboratory, keeping in mind the current travel and construction scenario in our country along with the need for upgraded tests in reference laboratories for identification up to species level remains paramount. 


\section{ACKNOWLEDGMENTS}

Declaration of Conflicting Interests: The authors declare that there is no conflict of interest regarding the publication of this paper.

Funding source: None declared.

\section{REFERENCES}

1. Aytac B, Sehitoglu I. A Rare Parasitic Infection in Turkey: Schistosomiasis. Case Report. Turk Patholoji Derg 2012; 28:175-177.

2. Kali A. Schistosome Infections: An Indian Perspective. J Clin Diag Res 2015; 9(2):DE01DE04.

3. Parija CS. Textbook of Medical Parasitology. $4^{\text {th }}$ eds. Chennai -600 084. All India Publishers and Distributors 2013; 229-247.

4. Doudier B, Parola P, Dales PJ, Linzberger N, Brouqui $P$, Delmont J. Schistosomiasis as an unusual cause of appendicitis. Clin Microbiol Infect 2004;10(2):89-91.

5. VA Francolugo-Velez, J Zarzosa-Alguiar. Urinary tract infection from Schistosoma haematobium: a case in Cuernavaca, Morelos, Mexico. Rev Mex Urol 2010; 70(3):187-192.

6. Neal MP. Schistosomiasis-An Unusual Cause of Ureteral Obstruction. A Case History and Perspective. Clinical Medicine and Research 2004; 2(4):216-227.

7. Schebel MH, Elsayes MK, Abou MH, Elguindy MY, El-Diasty AT. Genitourinary Schistosomiasis: Life Cycle and Radiologic-Pathologic Findings. Radiographics 2012; 32:1031-1046.

8. Bamgbola FO. Urinary Schistosomiasis. Paediatr Nephrol 2014; 29:2113-3120.

9. Schanz A, Richter J, Beyer I, Baldus ES, Hess PA, Krussel SJ. Genital Schistosomiasis as a cause of female sterility and acute abdomen. Fertil Steril 2010; 93(6): 2075.

10. Aminu BM, Abdullahi K, Dattijo ML. Tubal Ectopic Gestation Associated with Genital Schistosomiasis: A Case Report. Afr J Reprod Health 2014; 18(2):14.

11. Oranusi C K, Nwofor A, Onyiaorah I V, Ukah C O. Schistosomal stricture of the ureter-diagnostic dilemma. Niger J Clin Pract 2011;14:495-8

12. Houmsou R, Kela S, Suleiman M, Ogidi J. Urine colour as a rapid assessment indicator in evaluating the prevalence of Schistosoma haematobium infection in two endemic areas of Benue StateNigeria .Internet J Trop Med 2008;6(1).

13. Bierman FWW, Wetsteyn CFMJ, Gool VT. Presentation and Diagnosis of Imported Schistosomiasis: Relevance of Eosinophilia,
Microscopy for Ova, and Serology. J Travel Med 2005; 12: 9-13.

14. Meurs L, Mbow M, Polman K. Bladder Morbidity and Hepatic Fibrosis in Mixed Schistosoma haematobium and $S$. mansoni Infections: A Population-Wide Study in Northern Senegal. PLos Negl Trop Dis 2012; 6(9):e1829.

15. Da Silva LC, Chieffi PP, Carrilho FJ. Schistosoma mansoni-Clinical features. Gastroenterolgia y Hepatologia 2005; 28(1):30-39.

16. Agrawal CM, Rao GV. Indian Schistosomes: A Need for Further Investigations. J Parasitol Res 2011:250868. doi: 10.1155/2011/250868. 